\title{
Quantum trajectories for environment in superposition of coherent states
}

\author{
Anita Magdalena Dąbrowska ${ }^{1}$
}

Received: 20 December 2018 / Accepted: 21 May 2019 / Published online: 29 May 2019

(c) The Author(s) 2019

\begin{abstract}
We derive stochastic master equations for a quantum system interacting with a Bose field prepared in a superposition of continuous-mode coherent states. To determine a conditional evolution of the quantum system, we use a collision model with an environment given as an infinite chain of not interacting between themselves qubits prepared initially in an entangled state being a discrete analogue of a superposition of coherent states of the Bose field. The elements of the environment chain interact with the quantum system in turn one by one, and they are subsequently measured. We determine a conditional evolution of the quantum system for continuous in time observations of the output field as a limit of discrete recurrence equations. We consider the stochastic master equations for a counting as well as for a diffusive stochastic process.
\end{abstract}

Keywords Quantum trajectories · Quantum filtering · Stochastic master equation · Quantum non-Markovian dynamics · Collision model · Quantum stochastic calculus

\section{Introduction}

Quantum filtering theory [1-8] formulated within the framework of quantum stochastic Itô calculus (QSC) [9,10] gives the best state estimation of an open quantum system on the basis of a continuous in time measurement preformed on the Bose field interacting with the system. The filtering theory is formulated with making use of input-output formalism [11] wherein the input field is interpreted as the field before interaction with the system and the output field is interpreted as the field after this interaction. Information about the quantum system is gained in an indirect way by performing the measurements on the output field. In general, there are two types of

Anita Magdalena Dąbrowska

amdabrowska@gmail.com

1 Collegium Medicum Bydgoszcz, Nicolaus Copernicus University in Toruń, ul. Jagiellońska 15, 85-067 Bydgoszcz, Poland 
the measurement considered in the filtering theory, namely the photon counting and homodyne/heterodyne measurements which corresponds, respectively, to the counting and diffusion stochastic processes [6]. Evolution of an open quantum system conditioned on the results of the continuous in time measurement of the output field is given by the stochastic master equation called also in the literature the quantum filtering equation. The conditional state, depending on all past results of the measurement, creates quantum trajectory. By taking the average over all possible outcomes of the measurements, we get from the a posteriori evolution the a priori evolution given by the master equation. Clearly, the form of the filtering equation depends on the initial state of the environment and on the type of measurement performed on the output field. There exist many derivations of the filtering equations (see, for example, [1$4,8,12-15])$. One can find the rigorous derivations of the conditional evolution for the case when the Bose field is prepared in the Gaussian state, for instance, in [16-21]. The standard methods of determination of the filtering equation stop working when the Bose field is prepared in non-classical state. The initial temporal correlations in the Bose field make then the evolution of open system non-Markovian. The system becomes entangled with the environment, and its evolution is no longer given by one equation but by a set of equations. In this case to determine the conditional evolution of the system, one can apply a cascaded approach [7] with an ancilla system being a source of non-classical signal. The methods of determination of the filtering equation based on the idea of enlarging the Hilbert space of the compound system by the Hilbert space of ancilla were used for single-photon state in [22-25], for a Fock state in [26-28], and for a superposition of coherent states in [22,24]. Note, however, that ancilla system serves here only as a convenient theoretical mathematical device allowing to solve the problem of determination of the conditional evolution. Unfortunately, by introducing such auxiliary system we loose some physical intuition and the interpretation of quantum trajectories becomes thereby more difficult.

In the paper, we present derivation of the filtering equations for the environment prepared in a superposition of coherent states. Instead of the methods based on the concept of ancilla and QSC, we use quantum repeating interactions and measurements model [29-32], known also in the physical literature as a collision model [33]. We consider the environment modeled by an infinite chain of qubits which interact in turn one by one with a quantum system. After each interaction, the measurement is preformed on the last qubit interacted with the system. The essential properties of our model are that each qubit interacts with the system only once and that the environment qubits do not interact between themselves. So in the paper we use the toy Fock space as an approximation of the symmetrical Fock space [15,29,34,38-41]. The idea of obtaining the differential filtering equations from difference equations was implemented for the Markovian case in [14,15,34,35]. As shown in [36,37], it can be successfully applied also for the non-Markovian case.

The paper is organized as follows. In Sect. 2, we introduce a description of the environment and its interaction with the quantum system. Section 3 is devoted to derivation of the conditional evolution of open system for the case when the environment is prepared in a coherent state. In Sect. 4 the conditional evolution of open system for the bath in a superposition of coherent states is investigated. As an example, we present 
the a priori and the a posteriori dynamics of a single mode cavity in Sect. 5. Our results are briefly summarized in Sect. 6.

\section{The unitary system and environment evolution}

Let us consider a quantum system $\mathcal{S}$ of the Hilbert space $\mathcal{H}_{\mathcal{S}}$ interacting with an environment consisting of a sequence of qubits. We assume that the environment qubits do not interact between themselves, but they interact in a successive way with the system $\mathcal{S}$ each during the time interval of the length $\tau$. At a given moment $\mathcal{S}$ interacts with only one of the environment qubits. The Hilbert space of the environment is

$$
\mathcal{H}_{\mathcal{E}}=\bigotimes_{k=0}^{+\infty} \mathcal{H}_{\mathcal{E}, k}
$$

where $\mathcal{H}_{\mathcal{E}, k}$ stands for the Hilbert space of the $k$-th qubit interacting with $\mathcal{S}$ in the time interval $[k \tau,(k+1) \tau)$. We start from a discrete in time model of repeated interactions (collisions) to show finally its limit with time treated as a continuous variable. We will treat $\tau$ as a small time and work to linear order in $\tau$ (we neglect all higher order terms in $\tau$ ).

We assume that the unitary evolution of the compound $\mathcal{E}+\mathcal{S}$ system is governed by $[29,31]$

$$
U_{j}=\mathbb{V}_{j-1} \mathbb{V}_{j-2} \ldots \mathbb{V}_{0} \text { for } j \geq 1, \quad U_{0}=\mathbb{1}
$$

where $\mathbb{V}_{k}$ is the unitary operator acting non-trivially only in the Hilbert space $\mathcal{H}_{\mathcal{E}, k} \otimes$ $\mathcal{H}_{S}$, that is,

$$
\mathbb{V}_{k}=\bigotimes_{i=0}^{k-1} \mathbb{1}_{i} \otimes V_{k}
$$

and

$$
V_{k}=\exp \left(-i \tau H_{k}\right)
$$

with

$$
H_{k}=\bigotimes_{i=k}^{+\infty} \mathbb{1}_{i} \otimes H_{\mathcal{S}}+\frac{i}{\sqrt{\tau}}\left(\sigma_{k}^{+} \otimes \bigotimes_{i=k+1}^{+\infty} \mathbb{1}_{i} \otimes L-\sigma_{k}^{-} \otimes \bigotimes_{i=k+1}^{+\infty} \mathbb{1}_{i} \otimes L^{\dagger}\right)
$$

where $H_{\mathcal{S}}$ is the Hamiltonian of $\mathcal{S}, L$ is a bounded operator of $\mathcal{S}$, and $\sigma_{k}^{+}=|1\rangle_{k}\langle 0|$, $\sigma_{k}^{-}=|0\rangle_{k}\langle 1|$, where by $|0\rangle_{k}$ and $|1\rangle_{k}$ we denoted, respectively, the ground and excited states of the $k$-th qubit. The Hamiltonian $H_{k}$ is written in the interaction picture eliminating the free evolution of the bath. One can find a detailed discussion on the physical 
assumptions leading to (5), for instance, in [33,35,42]. For simplicity, we set the Planck constant $\hbar=1$. Note that $U_{j}$ describes the $j$-th first interactions and it has trivial action on $\bigotimes_{k=j}^{+\infty} \mathcal{H}_{\mathcal{E}, k}$.

Let us define in $\mathcal{H}_{\mathcal{E}, k}$ the vector $\left|\alpha_{k}\right\rangle_{k}$ by the formula [35]

$$
\left|\alpha_{k}\right\rangle_{k}=\mathrm{e}^{\sqrt{\tau}\left(\alpha_{k} \sigma_{k}^{+}-\alpha_{k}^{*} \sigma_{k}^{-}\right)}|0\rangle_{k}
$$

where $\alpha_{k} \in \mathbb{C}$. One can check that

$$
\left|\alpha_{k}\right\rangle_{k}=\left(1-\frac{\left|\alpha_{k}\right|^{2}}{2} \tau\right)|0\rangle_{k}+\alpha_{k} \sqrt{\tau}|1\rangle_{k}+O\left(\tau^{3 / 2}\right)
$$

and

$$
\left\langle\alpha_{k}\left|\sigma_{k}^{-}\right| \alpha_{k}\right\rangle=\sqrt{\tau} \alpha_{k}+O\left(\tau^{3 / 2}\right), \quad\left\langle\alpha_{k}\left|\sigma_{k}^{+} \sigma_{k}^{-}\right| \alpha_{k}\right\rangle=\tau\left|\alpha_{k}\right|^{2}+O\left(\tau^{2}\right)
$$

The coherent state in $\mathcal{H}_{\mathcal{E}}$ is defined as

$$
|\alpha\rangle=\bigotimes_{k=0}^{+\infty}\left|\alpha_{k}\right\rangle_{k}
$$

with the condition $\sum_{k=0}^{+\infty}\left|\alpha_{k}\right|^{2} \tau<\infty$.

Note that the vector state $|\alpha\rangle$ is a discrete analogue of coherent state defined in the symmetric Fock space considered in QSC. We will show that it allows in the continuous time limit to reproduce all results for the coherent state received within QSC.

\section{Quantum trajectories for a coherent state}

In this section we consider the case when the composed $\mathcal{E}+\mathcal{S}$ system is prepared initially in the pure product state

$$
|\alpha\rangle \otimes|\psi\rangle
$$

where $|\alpha\rangle$ is the coherent state of the environment.

\subsection{Photon counting}

We assume that after each interaction the measurement is performed on the last element of the environment chain just after its interaction with $\mathcal{S}$. A goal of this subsection is providing a description of the state of $\mathcal{S}$ conditioned on the results of the measurements of the observables

$$
\sigma_{k}^{-} \sigma_{k}^{+}=|1\rangle_{k}\langle 1|, \quad k=0,1,2, \ldots
$$


Theorem 1 The conditional state of $\mathcal{S}$ and the part of the environment which has not interacted with $\mathcal{S}$ up to $j \tau$ for the initial state (10) and the measurement of (11) at the moment $j \tau$ is given by

$$
\left|\tilde{\Psi}_{j}\right\rangle=\frac{\left|\Psi_{j}\right\rangle}{\sqrt{\left\langle\Psi_{j} \mid \Psi_{j}\right\rangle}},
$$

where

$$
\left|\Psi_{j}\right\rangle=\bigotimes_{k=j}^{+\infty}\left|\alpha_{k}\right\rangle_{k} \otimes\left|\psi_{j}\right\rangle
$$

and the conditional vector $\left|\psi_{j}\right\rangle$ from $\mathcal{H}_{S}$ satisfies the recurrence formula

$$
\left|\psi_{j+1}\right\rangle=M_{\eta_{j+1}}^{j}\left|\psi_{j}\right\rangle
$$

where $\eta_{j+1}$ stands for a random variable describing the $(j+1)$-th output of $(11)$, and $M_{\eta_{j+1}}^{j}$ has the form

$$
\begin{aligned}
& M_{0}^{j}=\mathbb{1}_{S}-\left(i H_{\mathcal{S}}+\frac{1}{2} L^{\dagger} L+L^{\dagger} \alpha_{j}+\frac{\left|\alpha_{j}\right|^{2}}{2}\right) \tau+O\left(\tau^{2}\right), \\
& M_{1}^{j}=\left(L+\alpha_{j}\right) \sqrt{\tau}+O\left(\tau^{3 / 2}\right) .
\end{aligned}
$$

Initially $\left|\psi_{j=0}\right\rangle=|\psi\rangle$ such that $\left|\tilde{\Psi}_{j=0}\right\rangle=|\alpha\rangle \otimes|\psi\rangle$.

It is clear that $\left|\tilde{\Psi}_{j}\right\rangle$ is the product state vector belonging to the Hilbert space $\bigotimes_{k=j}^{+\infty} \mathcal{H}_{\mathcal{E}, k} \otimes \mathcal{H}_{S}$. Note also that the conditional vector $\left|\psi_{j}\right\rangle$ depends on all results of the measurements performed on the bath qubits up to time $j \tau$.

Proof We prove the above theorem by an induction technique. So we start from the assumption that (13) holds and then check that

$$
\begin{aligned}
V_{j}\left|\Psi_{j}\right\rangle= & |0\rangle_{j} \otimes \bigotimes_{k=j+1}^{+\infty}\left|\alpha_{k}\right\rangle_{k} \otimes\left(\mathbb{1}_{S}-\left(i H_{\mathcal{S}}+\frac{1}{2} L^{\dagger} L+L^{\dagger} \alpha_{j}+\frac{\left|\alpha_{j}\right|^{2}}{2}\right) \tau\right. \\
& \left.+O\left(\tau^{2}\right)\right)\left|\psi_{j}\right\rangle \\
& +|1\rangle_{j} \otimes \bigotimes_{k=j+1}^{+\infty}\left|\alpha_{k}\right\rangle_{k} \otimes\left[\left(L+\alpha_{j}\right) \sqrt{\tau}+O\left(\tau^{3 / 2}\right)\right]\left|\psi_{j}\right\rangle .
\end{aligned}
$$

Now using the fact that the conditional vector $\left|\Psi_{j+1}\right\rangle$ from the Hilbert space $\bigotimes_{k=j+1}^{+\infty} \mathcal{H}_{\mathcal{E}, k} \otimes \mathcal{H}_{S}$ is defined by 


$$
\left(\Pi_{\eta_{j+1}}^{j} \otimes \bigotimes_{k=j+1}^{+\infty} \mathbb{1}_{k} \otimes \mathbb{1}_{S}\right) V_{j}\left|\Psi_{j}\right\rangle=\left|\eta_{j+1}\right\rangle_{j} \otimes\left|\Psi_{j+1}\right\rangle,
$$

where

$$
\Pi_{0}^{j}=|0\rangle_{j}\left\langle 0\left|, \quad \Pi_{1}^{j}=\right| 1\right\rangle_{j}\langle 1|,
$$

we readily find that $\left|\Psi_{j+1}\right\rangle$ has the form

$$
\left|\Psi_{j+1}\right\rangle=\bigotimes_{k=j+1}^{+\infty}\left|\alpha_{k}\right\rangle_{k} \otimes\left|\psi_{j+1}\right\rangle
$$

with $\left|\psi_{j+1}\right\rangle$ given by (14), which ends the proof.

\subsection{Homodyne detection}

Now we describe the evolution conditioned on the results of the measurements of the observables

$$
\sigma_{k}^{x}=\sigma_{k}^{+}+\sigma_{k}^{-}=|+\rangle_{k}\langle+|-|-\rangle_{k}\langle-|, \quad k=0,1,2, \ldots,
$$

where

$$
\begin{aligned}
|+\rangle_{k} & =\frac{1}{\sqrt{2}}\left(|0\rangle_{k}+|1\rangle_{k}\right), \\
|-\rangle_{k} & =\frac{1}{\sqrt{2}}\left(|0\rangle_{k}-|1\rangle_{k}\right),
\end{aligned}
$$

are vectors from the Hilbert space $\mathcal{H}_{\mathcal{E}, k}$.

Theorem 2 The conditional state of $\mathcal{S}$ and the part of the environment which has not interacted with $\mathcal{S}$ up to $j \tau$ for the initial state (10) and the measurement of (21) at the moment $j \tau$ is given by

$$
\left|\tilde{\Psi}_{j}\right\rangle=\frac{\left|\Psi_{j}\right\rangle}{\sqrt{\left\langle\Psi_{j} \mid \Psi_{j}\right\rangle}}
$$

where

$$
\left|\Psi_{j}\right\rangle=\bigotimes_{k=j}^{+\infty}\left|\alpha_{k}\right\rangle_{k} \otimes\left|\psi_{j}\right\rangle
$$

and the conditional vector $\left|\psi_{j}\right\rangle$ from $\mathcal{H}_{S}$ satisfies the recurrence formula

$$
\left|\psi_{j+1}\right\rangle=R_{\zeta_{j+1}}^{j}\left|\psi_{j}\right\rangle
$$


where $\zeta_{j+1}= \pm 1$ stands for a random variable describing $(j+1)$-th output of $(21)$, and

$$
\begin{aligned}
R_{\zeta_{j+1}}^{j}= & \frac{1}{\sqrt{2}}\left[\mathbb{1}_{S}-\left(i H_{\mathcal{S}}+\frac{1}{2} L^{\dagger} L+L^{\dagger} \alpha_{j}+\frac{\left|\alpha_{j}\right|^{2}}{2}\right) \tau\right. \\
& \left.+\left(L+\alpha_{j}\right) \zeta_{j+1} \sqrt{\tau}+O\left(\tau^{3 / 2}\right)\right] .
\end{aligned}
$$

Initially $\left|\psi_{j=0}\right\rangle=|\psi\rangle$ such that $\left|\tilde{\Psi}_{j=0}\right\rangle=|\alpha\rangle \otimes|\psi\rangle$.

Proof Assuming that (25) holds we get

$$
\begin{aligned}
V_{j}\left|\Psi_{j}\right\rangle= & \frac{1}{\sqrt{2}}|+\rangle_{j} \otimes \bigotimes_{k=j+1}^{+\infty}\left|\alpha_{k}\right\rangle_{k} \otimes\left\{\mathbb{1}_{S}-\left(i H_{\mathcal{S}}+\frac{1}{2} L^{\dagger} L+L^{\dagger} \alpha_{j}+\frac{\left|\alpha_{j}\right|^{2}}{2}\right) \tau\right. \\
& \left.+\left(L+\alpha_{j}\right) \sqrt{\tau}+O\left(\tau^{3 / 2}\right)\right\}\left|\psi_{j}\right\rangle \\
& +\frac{1}{\sqrt{2}}|-\rangle_{j} \otimes \bigotimes_{k=j+1}^{+\infty}\left|\alpha_{k}\right\rangle_{k} \otimes\left\{\mathbb{1}_{S}-\left(i H_{\mathcal{S}}+\frac{1}{2} L^{\dagger} L+L^{\dagger} \alpha_{j}+\frac{\left|\alpha_{j}\right|^{2}}{2}\right) \tau\right. \\
& \left.-\left(L+\alpha_{j}\right) \sqrt{\tau}+O\left(\tau^{3 / 2}\right)\right\}\left|\psi_{j}\right\rangle .
\end{aligned}
$$

The conditional vector $\left|\Psi_{j+1}\right\rangle$ from the Hilbert space $\bigotimes_{k=j+1}^{+\infty} \mathcal{H}_{\mathcal{E}, k} \otimes \mathcal{H}_{S}$ satisfies for the measurement of (21) the equation

$$
\left(\Pi_{\zeta_{j+1}}^{j} \otimes \bigotimes_{k=j+1}^{+\infty} \mathbb{1}_{k} \otimes \mathbb{1}_{S}\right) V_{j}\left|\Psi_{j}\right\rangle=\left|\zeta_{j+1}\right\rangle_{j} \otimes\left|\Psi_{j+1}\right\rangle
$$

where $\zeta_{j+1}$ has two possible values \pm 1 , and

$$
\Pi_{+1}^{j}=|+\rangle_{j}\left\langle+\left|, \quad \Pi_{-1}^{j}=\right|-\right\rangle_{j}\langle-| .
$$

It is seen that $\left|\Psi_{j+1}\right\rangle$ has the form of (25) and the vector $\left|\psi_{j}\right\rangle$ from $\mathcal{H}_{\mathcal{S}}$ satisfies the recurrence equation (27).

\section{Quantum trajectories for a superposition of coherent states}

Let us assume that the initial state of the compound $\mathcal{E}+\mathcal{S}$ system is given by

$$
\left(c_{\alpha}|\alpha\rangle+c_{\beta}|\beta\rangle\right) \otimes|\psi\rangle
$$

where $|\alpha\rangle$ and $|\beta\rangle$ are coherent states of $\mathcal{H}_{\mathcal{E}}$, and

$$
\left|c_{\alpha}\right|^{2}+c_{\alpha} c_{\beta}^{*}\langle\beta \mid \alpha\rangle+c_{\alpha}^{*} c_{\beta}\langle\alpha \mid \beta\rangle+\left|c_{\beta}\right|^{2}=1 .
$$

Note that in this case the bath qubits are prepared in an entangled state. 


\subsection{Photon counting}

Theorem 3 The conditional state of $\mathcal{S}$ and the part of the environment which has not interacted with $\mathcal{S}$ up to $j \tau$ for the initial state (31) and the measurement of (11) at the moment $j \tau$ is given by

$$
\left|\tilde{\Psi}_{j}\right\rangle=\frac{\left|\Psi_{j}\right\rangle}{\sqrt{\left\langle\Psi_{j} \mid \Psi_{j}\right\rangle}}
$$

where

$$
\left|\Psi_{j}\right\rangle=c_{\alpha} \bigotimes_{k=j}^{+\infty}\left|\alpha_{k}\right\rangle_{k} \otimes\left|\psi_{j}\right\rangle+c_{\beta} \bigotimes_{k=j}^{+\infty}\left|\beta_{k}\right\rangle_{k} \otimes\left|\varphi_{j}\right\rangle
$$

The conditional vectors $\left|\psi_{j}\right\rangle,\left|\varphi_{j}\right\rangle$ from $\mathcal{H}_{\mathcal{S}}$ in (34) are given by the recurrence formulas

$$
\begin{aligned}
\left|\psi_{j+1}\right\rangle & =M_{\eta_{j+1}}^{\alpha_{j}}\left|\psi_{j}\right\rangle, \\
\left|\varphi_{j+1}\right\rangle & =M_{\eta_{j+1}}^{\beta_{j}}\left|\varphi_{j}\right\rangle
\end{aligned}
$$

where $\eta_{j+1}=0,1$ stands for a random variable describing the $(j+1)$-th output of (11), and

$$
\begin{aligned}
& M_{0}^{\alpha_{j}}=\mathbb{1}_{S}-\left(i H_{\mathcal{S}}+\frac{1}{2} L^{\dagger} L+L^{\dagger} \alpha_{j}+\frac{\left|\alpha_{j}\right|^{2}}{2}\right) \tau+O\left(\tau^{2}\right), \\
& M_{0}^{\beta_{j}}=\mathbb{1}_{S}-\left(i H_{\mathcal{S}}+\frac{1}{2} L^{\dagger} L+L^{\dagger} \beta_{j}+\frac{\left|\beta_{j}\right|^{2}}{2}\right) \tau+O\left(\tau^{2}\right), \\
& M_{1}^{\alpha_{j}}=\left(L+\alpha_{j}\right) \sqrt{\tau}+O\left(\tau^{3 / 2}\right), \\
& M_{1}^{\beta_{j}}=\left(L+\beta_{j}\right) \sqrt{\tau}+O\left(\tau^{3 / 2}\right),
\end{aligned}
$$

and initially we have $\left|\psi_{0}\right\rangle=\left|\varphi_{0}\right\rangle=|\psi\rangle$.

Proof The proof is straightforward. We simply refer to the results of the previous Section and the linearity of the evolution of the total system.

Let us notice that the form of $\left|\Psi_{j}\right\rangle$ indicates that the system $\mathcal{S}$ becomes entangled with this part of the environment which has not interacted with $\mathcal{S}$ yet. Taking the partial trace of the operator $\left|\Psi_{j}\right\rangle\left\langle\Psi_{j}\right|$ over $\mathcal{S}$, we get the unnormalized state of the environment of the form

$$
\begin{aligned}
\rho_{j}^{\text {field }}= & \left|c_{\alpha}\right|^{2} \bigotimes_{k=j}^{+\infty}\left|\alpha_{k}\right\rangle_{k}\left\langle\alpha_{k}\left|\left\langle\psi_{j} \mid \psi_{j}\right\rangle+c_{\alpha} c_{\beta}^{*} \bigotimes_{k=j}^{+\infty}\right| \alpha_{k}\right\rangle_{k}\left\langle\beta_{k}\right|\left\langle\varphi_{j} \mid \psi_{j}\right\rangle \\
& +c_{\alpha}^{*} c_{\beta} \bigotimes_{k=j}^{+\infty}\left|\beta_{k}\right\rangle_{k}\left\langle\left.\alpha_{k}\left|\left\langle\psi_{j} \mid \varphi_{j}\right\rangle+\right| c_{\beta}\right|^{2}\left|\bigotimes_{k=j}^{+\infty}\right| \beta_{k}\right\rangle_{k}\left\langle\beta_{k}\right|\left\langle\varphi_{j} \mid \varphi_{j}\right\rangle
\end{aligned}
$$


The operator $\rho_{j}^{\text {field }}$ describes the conditional state of this part of the environment which has not interacted with $\mathcal{S}$ yet. It depends on all results of the measurements performed on the bath qubits up to $j \tau$. Therefore, we can say that the results of the measurements change our knowledge about the state of the future part of the environment.

In order to obtain the conditional state of $\mathcal{S}$, one has to take the partial trace of $\left|\tilde{\Psi}_{j}\right\rangle\left\langle\tilde{\Psi}_{j}\right|$ over the environment. One can check that the a posteriori state of $\mathcal{S}$ at the time $j \tau$ has the form

$$
\tilde{\rho}_{j}=\frac{\rho_{j}}{\operatorname{Tr} \rho_{j}}
$$

where

$$
\begin{aligned}
\rho_{j}= & \left|c_{\alpha}\right|^{2}\left|\psi_{j}\right\rangle\left\langle\psi_{j}\left|+c_{\alpha} c_{\beta}^{*} \prod_{k=j}^{+\infty}\left\langle\beta_{k} \mid \alpha_{k}\right\rangle\right| \psi_{j}\right\rangle\left\langle\varphi_{j}\left|+c_{\alpha}^{*} c_{\beta} \prod_{k=j}^{+\infty}\left\langle\alpha_{k} \mid \beta_{k}\right\rangle\right| \varphi_{j}\right\rangle\left\langle\psi_{j}\right| \\
& +\left|c_{\beta}\right|^{2}\left|\varphi_{j}\right\rangle\left\langle\varphi_{j}\right|
\end{aligned}
$$

and $\operatorname{Tr} \rho_{j}$ is the probability of a particular trajectory.

To derive the set of recurrence equations describing the stochastic evolution of $\mathcal{S}$, it is convenient to write down the conditional state of $\mathcal{S}$ at $j \tau$ in the form

$$
\tilde{\rho}_{j}=\left|c_{\alpha}\right|^{2} \tilde{\rho}_{j}^{\alpha \alpha}+c_{\alpha} c_{\beta}^{*} \tilde{\rho}_{j}^{\alpha \beta}+c_{\alpha}^{*} c_{\beta} \tilde{\rho}_{j}^{\beta \alpha}+\left|c_{\beta}\right|^{2} \tilde{\rho}_{j}^{\beta \beta}
$$

where

$$
\begin{aligned}
\tilde{\rho}_{j}^{\alpha \alpha} & =\frac{1}{\operatorname{Tr} \rho_{j}}\left|\psi_{j}\right\rangle\left\langle\psi_{j}\right|, \\
\tilde{\rho}_{j}^{\alpha \beta} & =\frac{\prod_{k=j}^{+\infty}\left\langle\beta_{k} \mid \alpha_{k}\right\rangle_{k}}{\operatorname{Tr} \rho_{j}}\left|\psi_{j}\right\rangle\left\langle\varphi_{j}\right|, \\
\tilde{\rho}_{j}^{\beta \alpha} & =\frac{\prod_{k=j}^{+\infty}\left\langle\alpha_{k} \mid \beta_{k}\right\rangle_{k}}{\operatorname{Tr} \rho_{j}}\left|\varphi_{j}\right\rangle\left\langle\psi_{j}\right|, \\
\tilde{\rho}_{j}^{\beta \beta} & =\frac{1}{\operatorname{Tr} \rho_{j}}\left|\varphi_{j}\right\rangle\left\langle\varphi_{j}\right| .
\end{aligned}
$$

In our derivation, we will use several times the formula

$$
\prod_{k=j}^{+\infty}{ }_{k}\left\langle\beta_{k} \mid \alpha_{k}\right\rangle_{k}=\prod_{k=j+1}^{+\infty}{ }_{k}\left\langle\beta_{k} \mid \alpha_{k}\right\rangle_{k}\left(1-\frac{1}{2}\left(\left|\alpha_{j}\right|^{2}+\left|\beta_{j}\right|^{2}-2 \alpha_{j} \beta_{j}^{*}\right) \tau+O\left(\tau^{2}\right)\right)
$$


following from

$$
{ }_{k}\left\langle\beta_{k} \mid \alpha_{k}\right\rangle_{k}=1-\frac{1}{2}\left(\left|\alpha_{k}\right|^{2}+\left|\beta_{k}\right|^{2}-2 \alpha_{k} \beta_{k}^{*}\right) \tau+O\left(\tau^{2}\right)
$$

Let us notice first that the conditional operator $\rho_{j+1}$ is given by the recurrence formula

$$
\begin{aligned}
\rho_{j+1}= & \left|c_{\alpha}\right|^{2} M_{\eta_{j+1}}^{\alpha_{j}}\left|\psi_{j}\right\rangle\left\langle\psi_{j}\left|M_{\eta_{j+1}}^{\alpha_{j} \dagger}+c_{\alpha} c_{\beta}^{*} \prod_{k=j+1}^{+\infty}\left\langle\beta_{k} \mid \alpha_{k}\right\rangle M_{\eta_{j+1}}^{\alpha_{j}}\right| \psi_{j}\right\rangle\left\langle\varphi_{j}\right| M_{\eta_{j+1}}^{\beta_{j} \dagger} \\
& +c_{\alpha}^{*} c_{\beta} \prod_{k=j+1}^{+\infty}\left\langle\alpha_{k} \mid \beta_{k}\right\rangle M_{\eta_{j+1}}^{\beta_{j}}\left|\varphi_{j}\right\rangle\left\langle\left.\psi_{j}\left|M_{\eta_{j+1}}^{\alpha_{j} \dagger}+\right| c_{\beta}\right|^{2} M_{\eta_{j+1}}^{\beta_{j}} \mid \varphi_{j}\right\rangle\left\langle\varphi_{j}\right| M_{\eta_{j+1}}^{\beta_{j} \dagger}
\end{aligned}
$$

where $\eta_{j+1}$ stands for the random variable having two possible values: 0 , 1 . Let us note that in order to determine $\tilde{\rho}_{j+1}$ we need to know the operators (45)-(48) at the moment $j \tau$ and the result of the next measurement. When the result of the measurement is 0 , then we obtain from Eqs. (35) and (36) the following set of discrete equations

$$
\begin{aligned}
\left|\psi_{j+1}\right\rangle\left\langle\psi_{j+1}\right|= & \left|\psi_{j}\right\rangle\left\langle\psi_{j}\right|-i\left[H_{\mathcal{S}},\left|\psi_{j}\right\rangle\left\langle\psi_{j}\right|\right] \tau-\frac{1}{2}\left\{L^{\dagger} L,\left|\psi_{j}\right\rangle\left\langle\psi_{j}\right|\right\} \tau \\
& -\left|\psi_{j}\right\rangle\left\langle\psi_{j}\left|L \alpha_{j}^{*} \tau-L^{\dagger}\right| \psi_{j}\right\rangle\left\langle\psi_{j}\left|\alpha_{j} \tau-\right| \psi_{j}\right\rangle\left\langle\left.\psi_{j}|| \alpha_{j}\right|^{2} \tau+O\left(\tau^{2}\right),\right. \\
\left|\psi_{j+1}\right\rangle\left\langle\varphi_{j+1}\right|= & \left|\psi_{j}\right\rangle\left\langle\varphi_{j}\right|\left(1-\frac{1}{2}\left(\left|\alpha_{j}\right|^{2}+\left|\beta_{j}\right|^{2}\right) \tau\right) \\
& -i\left[H_{\mathcal{S}},\left|\psi_{j}\right\rangle\left\langle\varphi_{j}\right|\right] \tau-\frac{1}{2}\left\{L^{\dagger} L,\left|\psi_{j}\right\rangle\left\langle\varphi_{j}\right|\right\} \tau \\
& -\left|\psi_{j}\right\rangle\left\langle\varphi_{j}\left|L \beta_{j}^{*} \tau-L^{\dagger}\right| \psi_{j}\right\rangle\left\langle\varphi_{j}\right| \alpha_{j} \tau+O\left(\tau^{2}\right), \\
\left|\varphi_{j+1}\right\rangle\left\langle\varphi_{j+1}\right|= & \left|\varphi_{j}\right\rangle\left\langle\varphi_{j}\right|-i\left[H_{\mathcal{S}},\left|\varphi_{j}\right\rangle\left\langle\varphi_{j}\right|\right] \tau-\frac{1}{2}\left\{L^{\dagger} L,\left|\varphi_{j}\right\rangle\left\langle\varphi_{j}\right|\right\} \tau \\
& -\left|\varphi_{j}\right\rangle\left\langle\varphi_{j}\left|L \beta_{j}^{*} \tau-L^{\dagger}\right| \varphi_{j}\right\rangle\left\langle\varphi_{j}\left|\beta_{j} \tau-\right| \varphi_{j}\right\rangle\left\langle\left.\varphi_{j}|| \beta_{j}\right|^{2} \tau+O\left(\tau^{2}\right) .\right.
\end{aligned}
$$

The conditional probability of the outcome 0 at the moment $(j+1) \tau$ when the a posteriori state of $\mathcal{S}$ at $j \tau$ was $\tilde{\rho}_{j}$ is defined as

$$
p_{j+1}\left(0 \mid \tilde{\rho}_{j}\right)=\frac{\operatorname{Tr} \rho_{j+1}}{\operatorname{Tr} \rho_{j}},
$$

where $\rho_{j+1}$ is given by (51) for $\eta_{j}=0$. Hence, we obtain the formula

$$
p_{j+1}\left(0 \mid \tilde{\rho}_{j}\right)=1-v_{j} \tau+O\left(\tau^{2}\right)
$$


where

$$
\begin{aligned}
v_{j} & =\left|c_{\alpha}\right|^{2} v_{j}^{\alpha \alpha}+c_{\alpha} c_{\beta}^{*} v_{j}^{\alpha \beta}+c_{\alpha}^{*} c_{\beta} v_{j}^{\beta \alpha}+\left|c_{\beta}\right|^{2} v_{j}^{\beta \beta}, \\
v_{j}^{\alpha \alpha} & =\operatorname{Tr}\left[\left(L^{\dagger} L+L \alpha_{j}^{*}+L^{\dagger} \alpha_{j}+\left|\alpha_{j}\right|^{2}\right) \tilde{\rho}_{j}^{\alpha \alpha}\right], \\
v_{j}^{\alpha \beta} & =\operatorname{Tr}\left[\left(L^{\dagger} L+L \beta_{j}^{*}+L^{\dagger} \alpha_{j}+\alpha_{j} \beta_{j}^{*}\right) \tilde{\rho}_{j}^{\alpha \beta}\right], \\
v_{j}^{\beta \alpha} & =\operatorname{Tr}\left[\left(L^{\dagger} L+L \alpha_{j}^{*}+L^{\dagger} \beta_{j}+\alpha_{j}^{*} \beta_{j}\right) \tilde{\rho}_{j}^{\beta \alpha}\right], \\
v_{j}^{\beta \beta} & =\operatorname{Tr}\left[\left(L^{\dagger} L+L \beta_{j}^{*}+L^{\dagger} \beta_{j}+\left|\beta_{j}\right|^{2}\right) \tilde{\rho}_{j}^{\beta \beta}\right] .
\end{aligned}
$$

Now, making use of the fact that

$$
\frac{1}{\operatorname{Tr} \rho_{j+1}}=\frac{1}{\operatorname{Tr} \rho_{j}}\left(1+v_{j} \tau+O\left(\tau^{2}\right)\right)
$$

we obtain the set of difference equations

$$
\begin{aligned}
\tilde{\rho}_{j+1}^{\alpha \alpha}-\tilde{\rho}_{j}^{\alpha \alpha}= & \tilde{\rho}_{j}^{\alpha \alpha} v_{j} \tau-i\left[H_{\mathcal{S}}, \tilde{\rho}_{j}^{\alpha \alpha}\right] \tau-\frac{1}{2}\left\{L^{\dagger} L, \tilde{\rho}_{j}^{\alpha \alpha}\right\} \tau-\tilde{\rho}_{j}^{\alpha \alpha} L \alpha_{j}^{*} \tau \\
& -L^{\dagger} \tilde{\rho}_{j}^{\alpha \alpha} \alpha_{j} \tau-\tilde{\rho}_{j}^{\alpha \alpha}\left|\alpha_{j}\right|^{2} \tau+O\left(\tau^{2}\right), \\
\tilde{\rho}_{j+1}^{\alpha \beta}-\tilde{\rho}_{j}^{\alpha \beta}= & \tilde{\rho}_{j}^{\alpha \beta} v_{j} \tau-i\left[H_{\mathcal{S}}, \tilde{\rho}_{j}^{\alpha \beta}\right] \tau-\frac{1}{2}\left\{L^{\dagger} L, \tilde{\rho}_{j}^{\alpha \beta}\right\} \tau-\tilde{\rho}_{j}^{\alpha \beta} L \beta_{j}^{*} \tau \\
& -L^{\dagger} \tilde{\rho}_{j}^{\alpha \beta} \alpha_{j} \tau-\tilde{\rho}_{j}^{\alpha \beta} \beta_{j}^{*} \alpha_{j} \tau+O\left(\tau^{2}\right), \\
\tilde{\rho}_{j+1}^{\beta \beta}-\tilde{\rho}_{j}^{\beta \beta}= & \tilde{\rho}_{j}^{\beta \beta} v_{j} \tau-i\left[H_{\mathcal{S}}, \tilde{\rho}_{j}^{\beta \beta}\right] \tau-\frac{1}{2}\left\{L^{\dagger} L, \tilde{\rho}_{j}^{\beta \beta}\right\} \tau-\tilde{\rho}_{j}^{\beta \beta} L \beta_{j}^{*} \tau \\
& -L^{\dagger} \tilde{\rho}_{j}^{\beta \beta} \beta_{j} \tau-\tilde{\rho}_{j}^{\beta \beta}\left|\beta_{j}\right|^{2} \tau+O\left(\tau^{2}\right) .
\end{aligned}
$$

One can get the equation for the operator $\tilde{\rho}_{j}^{\beta \alpha}$ using the fact that $\tilde{\rho}_{j}^{\beta \alpha}=\left(\tilde{\rho}_{j}^{\alpha \beta}\right)^{\dagger}$.

When the result of the measurement at the moment $(j+1) \tau$ is 1 , we get the following recurrence formulas

$$
\begin{aligned}
\left|\psi_{j+1}\right\rangle\left\langle\psi_{j+1}\right|= & \left(L\left|\psi_{j}\right\rangle\left\langle\psi_{j}\left|L^{\dagger}+L\right| \psi_{j}\right\rangle\left\langle\psi_{j}\right| \alpha_{j}^{*}\right. \\
& +\left|\psi_{j}\right\rangle\left\langle\psi_{j}\left|L^{\dagger} \alpha_{j}+\right| \psi_{j}\right\rangle\left\langle\left.\psi_{j}|| \alpha_{j}\right|^{2}\right) \tau+O\left(\tau^{2}\right), \\
\left|\psi_{j+1}\right\rangle\left\langle\varphi_{j+1}\right|= & \left(L\left|\psi_{j}\right\rangle\left\langle\varphi_{j}\left|L^{\dagger}+L\right| \psi_{j}\right\rangle\left\langle\varphi_{j}\right| \beta_{j}^{*}\right. \\
& \left.+\left|\psi_{j}\right\rangle\left\langle\varphi_{j}\left|L^{\dagger} \alpha_{j}+\right| \psi_{j}\right\rangle\left\langle\varphi_{j}\right| \alpha_{j} \beta_{j}^{*}\right) \tau+O\left(\tau^{2}\right), \\
\left|\varphi_{j+1}\right\rangle\left\langle\varphi_{j+1}\right|= & \left(L\left|\varphi_{j}\right\rangle\left\langle\varphi_{j}\left|L^{\dagger}+L\right| \varphi_{j}\right\rangle\left\langle\varphi_{j}\right| \beta_{j}^{*}\right. \\
& +\left|\varphi_{j}\right\rangle\left\langle\varphi_{j}\left|L^{\dagger} \beta_{j}+\right| \varphi_{j}\right\rangle\left\langle\left.\varphi_{j}|| \beta_{j}\right|^{2}\right) \tau+O\left(\tau^{2}\right) .
\end{aligned}
$$


The conditional probability of the outcome 1 at the moment $(j+1) \tau$ when the a posteriori state of $\mathcal{S}$ at the moment $j \tau$ was $\tilde{\rho}_{j}$ is defined by

$$
p_{j+1}\left(1 \mid \tilde{\rho}_{j}\right)=\frac{\operatorname{Tr} \rho_{j+1}}{\operatorname{Tr} \rho_{j}},
$$

where $\rho_{j+1}$ is given by (51) with $\eta_{j}=1$. One can check that

$$
p_{j+1}\left(1 \mid \tilde{\rho}_{j}\right)=v_{j} \tau+O\left(\tau^{2}\right)
$$

where the conditional intensity $v_{j}$ is defined by (57). So for the result 1 we find that

$$
\begin{aligned}
& \tilde{\rho}_{j+1}^{\alpha \alpha}=\frac{1}{v_{j}}\left(L \tilde{\rho}_{j}^{\alpha \alpha} L^{\dagger}+L \tilde{\rho}_{j}^{\alpha \alpha} \alpha_{j}^{*}+\tilde{\rho}_{j}^{\alpha \alpha} L^{\dagger} \alpha_{j}+\tilde{\rho}_{j}^{\alpha \alpha}\left|\alpha_{j}\right|^{2}\right)+O(\tau), \\
& \tilde{\rho}_{j+1}^{\alpha \beta}=\frac{1}{v_{j}}\left(L \tilde{\rho}_{j}^{\alpha \beta} L^{\dagger}+L \tilde{\rho}_{j}^{\alpha \beta} \beta_{j}^{*}+\tilde{\rho}_{j}^{\alpha \beta} L^{\dagger} \alpha_{j}+\tilde{\rho}_{j}^{\alpha \beta} \alpha_{j} \beta_{j}^{*}\right)+O(\tau), \\
& \tilde{\rho}_{j+1}^{\beta \beta}=\frac{1}{v_{j}}\left(L \tilde{\rho}_{j}^{\beta \beta} L^{\dagger}+L \tilde{\rho}_{j}^{\beta \beta} \beta_{j}^{*}+\tilde{\rho}_{j}^{\beta \beta} L^{\dagger} \beta_{j}+\tilde{\rho}_{j}^{\beta \beta}\left|\beta_{j}\right|^{2}\right)+O(\tau) .
\end{aligned}
$$

Let us introduce now the stochastic discrete process

$$
n_{j}=\sum_{k=0}^{j} \eta_{k}
$$

with the increment

$$
\Delta n_{j}=n_{j+1}-n_{j}
$$

One can check that the conditional expectation

$$
\mathbb{E}\left[\Delta n_{j} \mid \tilde{\rho}_{j}\right]=v_{j} \tau+O\left(\tau^{2}\right)
$$

Finally, by combining Eqs. (63)-(65) with Eqs. (71)-(73), we obtain the set of stochastic difference equations

$$
\begin{aligned}
\tilde{\rho}_{j+1}^{\alpha \alpha}-\tilde{\rho}_{j}^{\alpha \alpha}= & \mathcal{L} \tilde{\rho}_{j}^{\alpha \alpha} \tau+\left[\tilde{\rho}_{j}^{\alpha \alpha}, L^{\dagger}\right] \alpha_{j} \tau+\left[L, \tilde{\rho}_{j}^{\alpha \alpha}\right] \alpha_{j}^{*} \tau \\
& +\left\{\frac { 1 } { v _ { j } } \left(L \tilde{\rho}_{j}^{\alpha \alpha} L^{\dagger}+\tilde{\rho}_{j}^{\alpha \alpha} L^{\dagger} \alpha_{j}+L \tilde{\rho}_{j}^{\alpha \alpha} \alpha_{j}^{*}\right.\right. \\
& \left.\left.+\tilde{\rho}_{j}^{\alpha \alpha}\left|\alpha_{j}\right|^{2}\right)-\tilde{\rho}_{j}^{\alpha \alpha}\right\}\left(\Delta n_{j}-v_{j} \tau\right), \\
\tilde{\rho}_{j+1}^{\alpha \beta}-\tilde{\rho}_{j}^{\alpha \beta}= & \mathcal{L} \tilde{\rho}_{j}^{\alpha \beta} \tau+\left[\tilde{\rho}_{j}^{\alpha \beta}, L^{\dagger}\right] \alpha_{j} \tau+\left[L, \tilde{\rho}_{j}^{\alpha \beta}\right] \beta_{j}^{*} \tau \\
& +\left\{\frac { 1 } { v _ { j } } \left(L \tilde{\rho}_{j}^{\alpha \beta} L^{\dagger}+\tilde{\rho}_{j}^{\alpha \beta} L^{\dagger} \alpha_{j}+L \tilde{\rho}_{j}^{\alpha \beta} \beta_{j}^{*}\right.\right. \\
& \left.\left.+\tilde{\rho}_{j}^{\alpha \beta} \beta_{j}^{*} \alpha_{j}\right)-\tilde{\rho}_{j}^{\alpha \beta}\right\}\left(\Delta n_{j}-v_{j} \tau\right)
\end{aligned}
$$




$$
\begin{aligned}
\tilde{\rho}_{j+1}^{\beta \beta}-\tilde{\rho}_{j}^{\beta \beta}= & \mathcal{L} \tilde{\rho}_{j}^{\beta \beta} \tau+\left[\tilde{\rho}_{j}^{\beta \beta}, L^{\dagger}\right] \beta_{j} \tau+\left[L, \tilde{\rho}_{j}^{\beta \beta}\right] \beta_{j}^{*} \tau \\
& +\left\{\frac { 1 } { v _ { j } } \left(L \tilde{\rho}_{j}^{\beta \beta} L^{\dagger}+\tilde{\rho}_{j}^{\beta \beta} L^{\dagger} \beta_{j}+L \tilde{\rho}_{j}^{\beta \beta} \beta_{j}^{*}\right.\right. \\
& \left.\left.+\tilde{\rho}_{j}^{\beta \beta}\left|\beta_{t}\right|^{2}\right)-\tilde{\rho}_{j}^{\beta \beta}\right\}\left(\Delta n_{j}-v_{j} \tau\right),
\end{aligned}
$$

where

$$
\mathcal{L} \rho=-i\left[H_{\mathcal{S}}, \rho\right]-\frac{1}{2}\left\{L^{\dagger} L, \rho\right\}+L \rho L^{\dagger}
$$

and the initial condition $\tilde{\rho}_{0}^{\alpha \alpha}=\tilde{\rho}_{0}^{\beta \beta}=|\psi\rangle\left\langle\psi\left|, \tilde{\rho}_{0}^{\alpha \beta}=\langle\beta \mid \alpha\rangle\right| \psi\right\rangle\langle\psi|$. We dropped here all terms that do not contribute to the continuous time limit when $\tau \rightarrow d t$. Note that when $\Delta n_{j}$ is equal to 0, then Eqs. (77)-(79) reduce to Eqs. (63)-(65), and when $\Delta n_{j}$ is equal to 1, then all the terms proportional to $\tau$ in Eqs. (77)-(79) are negligible and we obtain the formulas (71)-(73).

Let us notice that to get the continuous in time evolution of $\mathcal{S}$, we fix time $t=j \tau$ such that when $j \rightarrow+\infty$, we have $\tau \rightarrow 0$. Of course, we take $t$ fixed but arbitrary. Thus, in the continuous time limit we get from (77)-(79) the set of the stochastic differential equations of the form

$$
\begin{aligned}
\mathrm{d} \tilde{\rho}_{t}^{\alpha \alpha}= & \mathcal{L} \tilde{\rho}_{t}^{\alpha \alpha} \mathrm{d} t+\left[\tilde{\rho}_{t}^{\alpha \alpha}, L^{\dagger}\right] \alpha_{t} \mathrm{~d} t+\left[L, \tilde{\rho}_{t}^{\alpha \alpha}\right] \alpha_{t}^{*} \mathrm{~d} t \\
& +\left\{\frac { 1 } { v _ { j } } \left(L \tilde{\rho}_{t}^{\alpha \alpha} L^{\dagger}+\tilde{\rho}_{t}^{\alpha \alpha} L^{\dagger} \alpha_{t}+L \tilde{\rho}_{t}^{\alpha \alpha} \alpha_{t}^{*}\right.\right. \\
& \left.\left.+\tilde{\rho}_{t}^{\alpha \alpha}\left|\alpha_{t}\right|^{2}\right)-\tilde{\rho}_{t}^{\alpha \alpha}\right\}\left(\mathrm{d} n_{t}-v_{t} \mathrm{~d} t\right), \\
\mathrm{d} \tilde{\rho}_{t}^{\alpha \beta}= & \mathcal{L} \tilde{\rho}_{t}^{\alpha \beta} \mathrm{d} t+\left[\tilde{\rho}_{t}^{\alpha \beta}, L^{\dagger}\right] \alpha_{t} \mathrm{~d} t+\left[L, \tilde{\rho}_{t}^{\alpha \beta}\right] \beta_{t}^{*} \mathrm{~d} t \\
& +\left\{\frac { 1 } { v _ { t } } \left(L \tilde{\rho}_{t}^{\alpha \beta} L^{\dagger}+\tilde{\rho}_{t}^{\alpha \beta} L^{\dagger} \alpha_{t}+L \tilde{\rho}_{t}^{\alpha \beta} \beta_{t}^{*}\right.\right. \\
& \left.\left.+\tilde{\rho}_{t}^{\alpha \beta} \beta_{t}^{*} \alpha_{t}\right)-\tilde{\rho}_{t}^{\alpha \beta}\right\}\left(\mathrm{d} n_{t}-v_{t} \mathrm{~d} t\right) \\
\mathrm{d} \tilde{\rho}_{t}^{\beta \beta}= & \mathcal{L} \tilde{\rho}_{t}^{\beta \beta} \mathrm{d} t+\left[\tilde{\rho}_{t}^{\beta \beta}, L^{\dagger}\right] \beta_{t} \mathrm{~d} t+\left[L, \tilde{\rho}_{t}^{\beta \beta}\right] \beta_{t}^{*} \mathrm{~d} t \\
& +\left\{\frac { 1 } { v _ { t } } \left(L \tilde{\rho}_{t}^{\beta \beta} L^{\dagger}+\tilde{\rho}_{t}^{\beta \beta} L^{\dagger} \beta_{t}+L \tilde{\rho}_{t}^{\beta \beta} \beta_{j}^{*}\right.\right. \\
& \left.\left.+\tilde{\rho}_{t}^{\beta \beta}\left|\beta_{t}\right|^{2}\right)-\tilde{\rho}_{t}^{\beta \beta}\right\}\left(\mathrm{d} n_{t}-v_{t} \mathrm{~d} t\right)
\end{aligned}
$$

and initially $\tilde{\rho}_{0}^{\alpha \alpha}=\tilde{\rho}_{0}^{\beta \beta}=|\psi\rangle\left\langle\psi\left|, \tilde{\rho}_{0}^{\alpha \beta}=\langle\beta \mid \alpha\rangle\right| \psi\right\rangle\langle\psi|$. The stochastic process $n_{t}$ is defined as the continuous limit of the discrete process $n_{j}$. The Itô table for $\mathrm{d} n_{t}$ is $\left(\mathrm{d} n_{t}\right)^{2}=\mathrm{d} n_{t}$ (we can measure at most one photon in the interval of length $d t$ ) and $\mathbb{E}\left[\mathrm{d} n_{t} \mid \tilde{\rho}_{t}\right]=v_{t} \mathrm{~d} t$, where 


$$
\begin{aligned}
v_{t} & =\left|c_{\alpha}\right|^{2} v_{t}^{\alpha \alpha}+c_{\alpha} c_{\beta}^{*} v_{t}^{\alpha \beta}+c_{\alpha}^{*} c_{\beta} v_{t}^{\beta \alpha}+\left|c_{\beta}\right|^{2} v_{t}^{\beta \beta}, \\
v_{t}^{\alpha \alpha} & =\operatorname{Tr}\left[\left(L^{\dagger} L+L \alpha_{t}^{*}+L^{\dagger} \alpha_{t}+\left|\alpha_{t}\right|^{2}\right) \tilde{\rho}_{t}^{\alpha \alpha}\right], \\
v_{t}^{\alpha \beta} & =\operatorname{Tr}\left[\left(L^{\dagger} L+L \beta_{t}^{*}+L^{\dagger} \alpha_{t}+\alpha_{t} \beta_{t}^{*}\right) \tilde{\rho}_{t}^{\alpha \beta}\right], \\
v_{t}^{\beta \alpha} & =\operatorname{Tr}\left[\left(L^{\dagger} L+L \alpha_{t}^{*}+L^{\dagger} \beta_{t}+\alpha_{t}^{*} \beta_{t}\right) \tilde{\rho}_{t}^{\beta \alpha}\right], \\
v_{t}^{\beta \beta} & =\operatorname{Tr}\left[\left(L^{\dagger} L+L \beta_{t}^{*}+L^{\dagger} \beta_{t}+\left|\beta_{t}\right|^{2}\right) \tilde{\rho}_{t}^{\beta \beta}\right] .
\end{aligned}
$$

Moreover, the complex functions $\alpha_{t}$ and $\beta_{t}$ satisfy the conditions

$$
\int_{0}^{+\infty}\left|\alpha_{t}\right|^{2} d t<+\infty, \quad \int_{0}^{+\infty}\left|\beta_{t}\right|^{2} d t<+\infty
$$

and

$$
\langle\beta \mid \alpha\rangle=\exp \left\{-\frac{1}{2} \int_{0}^{+\infty}\left(\left|\alpha_{t}\right|^{2}+\left|\beta_{t}\right|^{2}-2 \alpha_{t} \beta_{t}^{*}\right) d t\right\} .
$$

Thus, the a posteriori state of $\mathcal{S}$ is given as

$$
\tilde{\rho}_{t}=\left|c_{\alpha}\right|^{2} \tilde{\rho}_{t}^{\alpha \alpha}+c_{\alpha} c_{\beta}^{*} \tilde{\rho}_{t}^{\alpha \beta}+c_{\alpha}^{*} c_{\beta} \tilde{\rho}_{t}^{\beta \alpha}+\left|c_{\beta}\right|^{2} \tilde{\rho}_{t}^{\beta \beta}
$$

where the conditional operators $\tilde{\rho}_{t}^{\alpha \alpha}, \tilde{\rho}_{t}^{\alpha \beta}, \tilde{\rho}_{t}^{\beta \beta}$ satisfy Eqs. (81)-(83), and $\tilde{\rho}_{t}^{\beta \alpha}=$ $\left(\tilde{\rho}_{t}^{\alpha \beta}\right)^{\dagger}$. One can check that $\operatorname{Tr} \tilde{\rho}_{t}=1$ for any $t \geq 0$. The equations (81)-(83) agree with the stochastic master equations derived in [23] (see Sec. IV in [23]).

When we take an average of $\tilde{\rho}_{t}$ over all realizations of the stochastic process $n_{t}$ (all possible outcomes), we get the a priori evolution of the system $\mathcal{S}$. One can check that the a priori state of $\mathcal{S}$ is described by

$$
\varrho_{t}=\left|c_{\alpha}\right|^{2} \varrho_{t}^{\alpha \alpha}+c_{\alpha} c_{\beta}^{*} \varrho_{j}^{\alpha \beta}+c_{\alpha}^{*} c_{\beta} \varrho_{t}^{\beta \alpha}+\left|c_{\beta}\right|^{2} \varrho_{t}^{\beta \beta},
$$

where the operators $\varrho_{t}^{\alpha \alpha}, \varrho_{t}^{\alpha \beta}, \varrho_{t}^{\beta \beta}$ satisfy the differential equations

$$
\begin{aligned}
& \dot{\varrho}_{t}^{\alpha \alpha}=\mathcal{L} \varrho_{t}^{\alpha \alpha}+\left[\varrho_{t}^{\alpha \alpha}, L^{\dagger}\right] \alpha_{t}+\left[L, \varrho_{t}^{\alpha \alpha}\right] \alpha_{t}^{*}, \\
& \dot{\varrho}_{t}^{\alpha \beta}=\mathcal{L} \varrho_{t}^{\alpha \beta}+\left[\varrho_{t}^{\alpha \beta}, L^{\dagger}\right] \alpha_{t}+\left[L, \varrho_{t}^{\alpha \beta}\right] \beta_{t}^{*}, \\
& \dot{\varrho}_{t}^{\beta \beta}=\mathcal{L} \varrho_{t}^{\beta \beta}+\left[\varrho_{t}^{\beta \beta}, L^{\dagger}\right] \beta_{t}+\left[L, \varrho_{t}^{\beta \beta}\right] \beta_{t}^{*},
\end{aligned}
$$

where $\mathcal{L}$ acts as (80). The initial condition is $\varrho_{0}^{\alpha \alpha}=\varrho_{0}^{\beta \beta}=|\psi\rangle\langle\psi|, \varrho_{0}^{\alpha \beta}=$ $\langle\beta \mid \alpha\rangle|\psi\rangle\langle\psi|$, and $\varrho_{t}^{\beta \alpha}=\left(\varrho_{t}^{\alpha \beta}\right)^{\dagger}$. One can easily check that $\operatorname{Tr} \varrho_{t}^{\alpha \alpha}=\operatorname{Tr} \varrho_{t}^{\beta \beta}=1$, $\operatorname{Tr} \varrho_{t}^{\alpha \beta}=\langle\beta \mid \alpha\rangle$, and $\operatorname{Tr} \varrho_{t}^{\beta \alpha}=\langle\alpha \mid \beta\rangle$ for any $t \geq 0$. 


\subsection{Homodyne detection}

Theorem 4 The conditional state of $\mathcal{S}$ and the part of the environment which has not interacted with $\mathcal{S}$ up to $j \tau$ for the initial state (31) and the measurement of (21) at the moment $j \tau$ is given by

$$
\left|\tilde{\Psi}_{j}\right\rangle=\frac{\left|\Psi_{j}\right\rangle}{\sqrt{\left\langle\Psi_{j} \mid \Psi_{j}\right\rangle}}
$$

where

$$
\left|\Psi_{j}\right\rangle=c_{\alpha} \bigotimes_{k=j}^{+\infty}\left|\alpha_{k}\right\rangle_{k} \otimes\left|\psi_{j}\right\rangle+c_{\beta} \bigotimes_{k=j}^{+\infty}\left|\beta_{k}\right\rangle_{k} \otimes\left|\varphi_{j}\right\rangle
$$

The conditional vectors $\left|\psi_{j}\right\rangle,\left|\varphi_{j}\right\rangle$ from $\mathcal{H}_{\mathcal{S}}$ in (34) are given by the recurrence formulas

$$
\begin{aligned}
\left|\psi_{j+1}\right\rangle & =R_{\zeta_{j+1}}^{\alpha_{j}}\left|\psi_{j}\right\rangle \\
\left|\varphi_{j+1}\right\rangle & =R_{\zeta_{j+1}}^{\beta_{j}}\left|\varphi_{j}\right\rangle
\end{aligned}
$$

where $\zeta_{j+1}$ stands for a random variable describing the $(j+1)$-th output of $(21)$, and

$$
\begin{aligned}
R_{\zeta_{j+1}}^{\alpha_{j}}= & \frac{1}{\sqrt{2}}\left[\mathbb{1}_{S}-\left(i H_{\mathcal{S}}+\frac{1}{2} L^{\dagger} L+L^{\dagger} \alpha_{j}+\frac{\left|\alpha_{j}\right|^{2}}{2}\right) \tau\right. \\
& \left.+\left(L+\alpha_{j}\right) \zeta_{j+1} \sqrt{\tau}+O\left(\tau^{3 / 2}\right)\right], \\
R_{\zeta_{j+1}}^{\beta_{j}}= & \frac{1}{\sqrt{2}}\left[\mathbb{1}_{S}-\left(i H_{\mathcal{S}}+\frac{1}{2} L^{\dagger} L+L^{\dagger} \beta_{j}+\frac{\left|\beta_{j}\right|^{2}}{2}\right) \tau\right. \\
& \left.+\left(L+\beta_{j}\right) \zeta_{j+1} \sqrt{\tau}+O\left(\tau^{3 / 2}\right)\right],
\end{aligned}
$$

and initially we have $\left|\psi_{0}\right\rangle=\left|\varphi_{0}\right\rangle=|\psi\rangle$.

Proof To prove Theorem (4), we use the result of Sect. 3.2 and the linearity of the evolution equation for the total system.

Clearly, the conditional state of $\mathcal{S}$ at the moment $j \tau$ has the form (44). We start derivation of the filtering equations for the stochastic operators (45)-(48) from writing down the recursive formulas

$$
\begin{aligned}
2\left|\psi_{j+1}\right\rangle\left\langle\psi_{j+1}\right|= & \left|\psi_{j}\right\rangle\left\langle\psi_{j}|+\mathcal{L}| \psi_{j}\right\rangle\left\langle\psi_{j}\right| \tau \\
& +\left[\left|\psi_{j}\right\rangle\left\langle\psi_{j}\right|, L^{\dagger}\right] \alpha_{j} \tau+\left[L,\left|\psi_{j}\right\rangle\left\langle\psi_{j}\right|\right] \alpha_{j}^{*} \tau \\
& +\left[\left(L+\alpha_{j}\right)\left|\psi_{j}\right\rangle\left\langle\psi_{j}|+| \psi_{j}\right\rangle\left\langle\psi_{j}\right|\left(L^{\dagger}+\alpha_{j}^{*}\right)\right] \zeta_{j+1} \sqrt{\tau},
\end{aligned}
$$




$$
\begin{aligned}
2\left|\psi_{j+1}\right\rangle\left\langle\varphi_{j+1}\right|= & \left|\psi_{j}\right\rangle\left\langle\varphi_{j}\right|\left(1-\frac{1}{2}\left(\left|\alpha_{j}\right|^{2}+\left|\beta_{j}\right|^{2}-2 \beta_{j}^{*} \alpha_{j}\right) \tau\right) \\
& +\mathcal{L}\left|\psi_{j+1}\right\rangle\left\langle\varphi_{j+1}\right| \tau \\
& +\left[\left|\psi_{j}\right\rangle\left\langle\varphi_{j}\right|, L^{\dagger}\right] \alpha_{j} \tau+\left[L,\left|\psi_{j}\right\rangle\left\langle\varphi_{j}\right|\right] \beta_{j}^{*} \tau \\
& +\left[\left(L+\alpha_{j}\right)\left|\psi_{j}\right\rangle\left\langle\varphi_{j}|+| \psi_{j}\right\rangle\left\langle\varphi_{j}\right|\left(L^{\dagger}+\beta_{j}^{*}\right)\right] \zeta_{j+1} \sqrt{\tau}, \\
2\left|\varphi_{j+1}\right\rangle\left\langle\varphi_{j+1}\right|= & \left|\varphi_{j}\right\rangle\left\langle\varphi_{j}|+\mathcal{L}| \varphi_{j}\right\rangle\left\langle\varphi_{j}\right| \tau \\
& +\left[\left|\varphi_{j}\right\rangle\left\langle\varphi_{j}\right|, L\right] \beta_{j} \mathrm{~d} t+\left[L,\left|\varphi_{j}\right\rangle\left\langle\varphi_{j}\right|\right] \beta_{j}^{*} \tau \\
& +\left[\left(L+\beta_{j}\right)\left|\varphi_{j}\right\rangle\left\langle\varphi_{j}|+| \varphi_{j}\right\rangle\left\langle\varphi_{j}\right|\left(L^{\dagger}+\beta_{j}^{*}\right)\right] \zeta_{j+1} \sqrt{\tau},
\end{aligned}
$$

We can readily deduce that the conditional probability of the result $\zeta_{j+1}$ at the moment $(j+1) \tau$ when the conditional state of $\mathcal{S}$ is $\tilde{\rho}_{j}$ at the time $j \tau$ is given by

$$
p_{j+1}\left(\zeta_{j+1} \mid \tilde{\rho}_{j}\right)=\frac{1}{2}\left(1+\mu_{j} \zeta_{j+1} \sqrt{\tau}\right)+O\left(\tau^{3 / 2}\right)
$$

where

$$
\mu_{j}=\left|c_{\alpha}\right|^{2} \mu_{j}^{\alpha \alpha}+c_{\alpha} c_{\beta}^{*} \mu_{j}^{\alpha \beta}+c_{\alpha}^{*} c_{\beta} \mu_{j}^{\beta \alpha}+\left|c_{\beta}\right|^{2} \mu_{j}^{\beta \beta}
$$

and

$$
\begin{aligned}
& \mu_{j}^{\alpha \alpha}=\operatorname{Tr}\left[\left(L+L^{\dagger}+\alpha_{j}+\alpha_{j}^{*}\right) \tilde{\rho}_{j}^{\alpha \alpha}\right], \\
& \mu_{j}^{\alpha \beta}=\operatorname{Tr}\left[\left(L+L^{\dagger}+\alpha_{j}+\beta_{j}^{*}\right) \tilde{\rho}_{j}^{\alpha \beta}\right], \\
& \mu_{j}^{\beta \alpha}=\operatorname{Tr}\left[\left(L+L^{\dagger}+\beta_{j}+\alpha_{j}^{*}\right) \tilde{\rho}_{j}^{\beta \alpha}\right], \\
& \mu_{j}^{\beta \beta}=\operatorname{Tr}\left[\left(L+L^{\dagger}+\beta_{j}+\beta_{j}^{*}\right) \tilde{\rho}_{j}^{\beta \beta}\right] .
\end{aligned}
$$

Thus for the discrete stochastic process $\zeta_{j}$ we obtain the conditional mean values

$$
\begin{aligned}
& \mathbb{E}\left[\zeta_{j+1} \mid \tilde{\rho}_{j}\right]=\mu_{j} \sqrt{\tau}+O\left(\tau^{3 / 2}\right), \\
& \mathbb{E}\left[\zeta_{j+1}^{2} \mid \tilde{\rho}_{j}\right]=1+O\left(\tau^{3 / 2}\right) .
\end{aligned}
$$

Let us introduce now the stochastic process

$$
q_{j}=\sqrt{\tau} \sum_{k=1}^{j} \zeta_{k}
$$

One can easily check that $\mathbb{E}\left[\Delta q_{j}=q_{j+1}-q_{j} \mid \tilde{\rho}_{j}\right]=\mu_{j} \tau+O\left(\tau^{3 / 2}\right)$. 
Now, taking into account that

$$
\frac{1}{\operatorname{Tr} \rho_{j+1}}=\frac{2}{\operatorname{Tr} \rho_{j}}\left(1-\mu_{j} \zeta_{j+1} \sqrt{\tau}+\mu_{j}^{2} \tau\right)
$$

after some algebra we find the set of the stochastic difference equations

$$
\begin{aligned}
\tilde{\rho}_{j+1}^{\alpha \alpha}-\tilde{\rho}_{j}^{\alpha \alpha}= & \mathcal{L} \tilde{\rho}_{j}^{\alpha \alpha} \tau+\left[\tilde{\rho}_{j}^{\alpha \alpha}, L^{\dagger}\right] \alpha_{j} \tau+\left[L, \tilde{\rho}_{j}^{\alpha \alpha}\right] \alpha_{j}^{*} \tau \\
& +\left[\left(L+\alpha_{j}\right) \tilde{\rho}_{j}^{\alpha \alpha}+\tilde{\rho}_{j}^{\alpha \alpha}\left(L^{\dagger}+\alpha_{j}^{*}\right)-\mu_{j} \tilde{\rho}_{j}^{\alpha \alpha}\right]\left(\Delta q_{j}-\mu_{j} \tau\right), \\
\tilde{\rho}_{j+1}^{\alpha \beta}-\tilde{\rho}_{j}^{\alpha \beta}= & \mathcal{L} \tilde{\rho}_{j}^{\alpha \beta} \tau+\left[\tilde{\rho}_{j}^{\alpha \beta}, L^{\dagger}\right] \alpha_{j} \tau+\left[L, \tilde{\rho}_{j}^{\alpha \beta}\right] \beta_{j}^{*} \tau \\
& +\left[\left(L+\alpha_{j}\right) \tilde{\rho}_{j}^{\alpha \beta}+\tilde{\rho}_{j}^{\alpha \beta}\left(L^{\dagger}+\beta_{j}^{*}\right)-\mu_{j} \tilde{\rho}_{j}^{\alpha \beta}\right]\left(\Delta q_{j}-\mu_{j} \tau\right), \\
\tilde{\rho}_{j+1}^{\beta \beta}-\tilde{\rho}_{j}^{\beta \beta}= & \mathcal{L} \tilde{\rho}_{j}^{\beta \beta} \tau+\left[\tilde{\rho}_{j}^{\beta \beta}, L^{\dagger}\right] \beta_{j} \tau+\left[L, \tilde{\rho}_{j}^{\beta \beta}\right] \beta_{j}^{*} \tau \\
& +\left[\left(L+\beta_{j}\right) \tilde{\rho}_{j}^{\beta \beta}+\tilde{\rho}_{j}^{\beta \beta}\left(L^{\dagger}+\beta_{j}^{*}\right)-\mu_{j} \tilde{\rho}_{j}^{\beta \beta}\right]\left(\Delta q_{j}-\mu_{j} \tau\right)
\end{aligned}
$$

with the initial conditions $\tilde{\rho}_{0}^{\alpha \alpha}=|\psi\rangle\left\langle\psi\left|, \tilde{\rho}_{0}^{\beta \beta}=\right| \psi\right\rangle\left\langle\psi\left|, \tilde{\rho}_{0}^{\alpha \beta}=\langle\beta \mid \alpha\rangle\right| \psi\right\rangle\langle\psi|$. Then in the continuous time limit, we have

$$
\begin{aligned}
\mathrm{d} \tilde{\rho}_{t}^{\alpha \alpha}= & \mathcal{L} \tilde{\rho}_{t}^{\alpha \alpha} \mathrm{d} t+\left[\tilde{\rho}_{t}^{\alpha \alpha}, L^{\dagger}\right] \alpha_{t} \mathrm{~d} t+\left[L, \tilde{\rho}_{t}^{\alpha \alpha}\right] \alpha_{t}^{*} \mathrm{~d} t \\
& +\left[\left(L+\alpha_{t}\right) \tilde{\rho}_{t}^{\alpha \alpha}+\tilde{\rho}_{t}^{\alpha \alpha}\left(L^{\dagger}+\alpha_{t}^{*}\right)-\mu_{t} \tilde{\rho}_{t}^{\alpha \alpha}\right]\left(\mathrm{d} q_{t}-\mu_{t} \mathrm{~d} t\right) \\
\mathrm{d} \tilde{\rho}_{t}^{\alpha \beta}= & \mathcal{L} \tilde{\rho}_{t}^{\alpha \beta} \mathrm{d} t+\left[\tilde{\rho}_{t}^{\alpha \beta}, L^{\dagger}\right] \alpha_{t} \mathrm{~d} t+\left[L, \tilde{\rho}_{t}^{\alpha \beta}\right] \beta_{t}^{*} \mathrm{~d} t \\
& +\left[\left(L+\alpha_{t}\right) \tilde{\rho}_{t}^{\alpha \beta}+\tilde{\rho}_{t}^{\alpha \beta}\left(L^{\dagger}+\beta_{t}^{*}\right)-\mu_{t} \tilde{\rho}_{t}^{\alpha \beta}\right]\left(\mathrm{d} q_{t}-\mu_{t} \mathrm{~d} t\right), \\
\mathrm{d} \tilde{\rho}_{t}^{\beta \beta}= & \mathcal{L} \tilde{\rho}_{t}^{\beta \beta} \mathrm{d} t+\left[\tilde{\rho}_{t}^{\beta \beta}, L^{\dagger}\right] \beta_{t} \mathrm{~d} t+\left[L, \tilde{\rho}_{t}^{\beta \beta}\right] \beta_{t}^{*} \mathrm{~d} t \\
& +\left[\left(L+\beta_{t}\right) \tilde{\rho}_{t}^{\beta \beta}+\tilde{\rho}_{t}^{\beta \beta}\left(L^{\dagger}+\beta_{t}^{*}\right)-\mu_{t} \tilde{\rho}_{t}^{\beta \beta}\right]\left(\mathrm{d} q_{t}-\mu_{t} \mathrm{~d} t\right)
\end{aligned}
$$

where

$$
\mu_{t}=\left|c_{\alpha}\right|^{2} \mu_{t}^{\alpha \alpha}+c_{\alpha} c_{\beta}^{*} \mu_{t}^{\alpha \beta}+c_{\alpha}^{*} c_{\beta} \mu_{t}^{\beta \alpha}+\left|c_{\beta}\right|^{2} \mu_{t}^{\beta \beta}
$$

and

$$
\begin{aligned}
& \mu_{t}^{\alpha \alpha}=\operatorname{Tr}\left[\left(L+L^{\dagger}+\alpha_{t}+\alpha_{t}^{*}\right) \tilde{\rho}_{t}^{\alpha \alpha}\right], \\
& \mu_{t}^{\alpha \beta}=\operatorname{Tr}\left[\left(L+L^{\dagger}+\alpha_{t}+\beta_{t}^{*}\right) \tilde{\rho}_{t}^{\alpha \beta}\right], \\
& \mu_{t}^{\beta \alpha}=\operatorname{Tr}\left[\left(L+L^{\dagger}+\beta_{t}+\alpha_{t}^{*}\right) \tilde{\rho}_{t}^{\beta \alpha}\right], \\
& \mu_{t}^{\beta \beta}=\operatorname{Tr}\left[\left(L+L^{\dagger}+\beta_{t}+\beta_{t}^{*}\right) \tilde{\rho}_{t}^{\beta \beta}\right] .
\end{aligned}
$$


The process $q_{j}$ in the limit $\tau \rightarrow 0$ converges to the stochastic process $q_{t}$ with the conditional probability $\mathbb{E}\left[d q_{t}=q_{t+d t}-q_{t} \mid \tilde{\rho}_{t}\right]=\mu_{t} \mathrm{~d} t$.

\section{An example: a cavity mode}

One can read about the emergence of collision model in quantum optics, for instance, in $[33,42]$. To derive the discrete model of repeated interactions and measurements one starts from description of interaction of a quantum system with a Bose field propagating in only one direction, making the rotating wave approximation and taking the flat spectrum of the field. Then one passes to the interaction picture with respect to the free dynamics of the field and takes the Hamiltonian of the field in the frequency domain with the lower limit of integration extended to $-\infty$. The time coarse-graining model arises from division of the field into some probe segments. Lack of an interaction between the system and the output field means that the photons emitted by the system leave immediately the interaction region and cannot be reabsorbed. We describe here briefly the a priori and the a posteriori evolution of a cavity mode coupled to a propagating one-dimensional Bose field in a superposition of two coherent states. Thus, we have

$$
\begin{aligned}
H_{\mathcal{S}} & =\omega_{0} a^{\dagger} a, \\
L & =\sqrt{\Gamma} a,
\end{aligned}
$$

where $a$ stands for the annihilation operator, $\omega_{0}>0$, and $\Gamma$ is a positive coupling constant. We consider here the case when the harmonic oscillator is initially in the coherent state

$$
a|u\rangle=u|u\rangle
$$

Then, the solution to the set of the master equations can be written then in the form

$$
\varrho_{t}=\left|c_{\alpha}\right|^{2}\left|f_{t}\right\rangle\left\langle f_{t}\left|+c_{\alpha} c_{\beta}^{*} \frac{\langle\beta \mid \alpha\rangle}{\left\langle g_{t} \mid f_{t}\right\rangle}\right| f_{t}\right\rangle\left\langle g_{t}\left|+c_{\alpha}^{*} c_{\beta} \frac{\langle\alpha \mid \beta\rangle}{\left\langle f_{t} \mid g_{t}\right\rangle}\right| g_{t}\right\rangle\left\langle\left. f_{t}|+| c_{\beta}\right|^{2} \mid g_{t}\right\rangle\left\langle g_{t}\right|,
$$

where

$$
\left\langle g_{t} \mid f_{t}\right\rangle=\exp \left\{-\frac{1}{2}\left(\left|g_{t}\right|^{2}+\left|f_{t}\right|^{2}-2 g_{t}^{*} f_{t}\right)\right\}
$$

and $\left|f_{t}\right\rangle,\left|g_{t}\right\rangle$ are coherent states of the harmonic oscillator with the amplitudes satisfying the equations

$$
\dot{f_{t}}=-\left(\mathrm{i} \omega_{0}+\frac{\Gamma}{2}\right) f_{t}-\sqrt{\Gamma} \alpha_{t}
$$




$$
\dot{g}_{t}=-\left(\mathrm{i} \omega_{0}+\frac{\Gamma}{2}\right) g_{t}-\sqrt{\Gamma} \beta_{t},
$$

where one can easily recognize the damping and driving terms. Hence, we obtain

$$
\begin{aligned}
& f_{t}=\mathrm{e}^{-\left(\mathrm{i} \omega_{0}+\frac{\Gamma}{2}\right) t}\left(u-\sqrt{\Gamma} \int_{0}^{t} \mathrm{e}^{\left(\mathrm{i} \omega_{0}+\frac{\Gamma}{2}\right) s} \alpha_{s} \mathrm{~d} s\right) \\
& g_{t}=\mathrm{e}^{-\left(\mathrm{i} \omega_{0}+\frac{\Gamma}{2}\right) t}\left(u-\sqrt{\Gamma} \int_{0}^{t} \mathrm{e}^{\left(\mathrm{i} \omega_{0}+\frac{\Gamma}{2}\right) s} \beta_{s} \mathrm{~d} s\right) .
\end{aligned}
$$

The solution (129) one can check by inserting it into Eqs. (93)-(95). The conditional state of the system can be written as

$$
\begin{aligned}
\tilde{\rho}_{t}= & \left|c_{\alpha}\right|^{2} G_{t}^{\alpha \alpha}\left|f_{t}\right\rangle\left\langle f_{t}\left|+c_{\alpha} c_{\beta}^{*} \frac{\langle\beta \mid \alpha\rangle}{\left\langle g_{t} \mid f_{t}\right\rangle} G_{t}^{\alpha \beta}\right| f_{t}\right\rangle\left\langle g_{t}\left|+c_{\alpha}^{*} c_{\beta} \frac{\langle\alpha \mid \beta\rangle}{\left\langle f_{t} \mid g_{t}\right\rangle} G_{t}^{\beta \alpha}\right| g_{t}\right\rangle\left\langle f_{t}\right| \\
& +\left|c_{\beta}\right|^{2} G_{t}^{\beta \beta}\left|g_{t}\right\rangle\left\langle g_{t}\right|,
\end{aligned}
$$

where the conditional amplitudes $f_{t}$ and $g_{t}$ coincide with the a priori ones given by (133) and (134), and the stochastic coefficients $G_{t}^{\alpha \alpha}, G_{t}^{\alpha \beta}, G_{t}^{\beta \alpha}$, and $G_{t}^{\beta \beta}$ for the counting stochastic process satisfy the equations

$$
\begin{aligned}
\mathrm{d} G_{t}^{\alpha \alpha} & =\left(\frac{v_{t}^{\alpha \alpha}}{v_{t}}-G_{t}^{\alpha \alpha}\right)\left(\mathrm{d} n_{t}-v_{t} \mathrm{~d} t\right), \\
\mathrm{d} G_{t}^{\alpha \beta} & =\left(\frac{v_{t}^{\alpha \beta}}{v_{t}\langle\beta \mid \alpha\rangle}-G_{t}^{\alpha \beta}\right)\left(\mathrm{d} n_{t}-v_{t} \mathrm{~d} t\right), \\
\mathrm{d} G_{t}^{\beta \alpha} & =\left(\frac{v_{t}^{\beta \alpha}}{v_{t}\langle\alpha \mid \beta\rangle}-G_{t}^{\beta \alpha}\right)\left(\mathrm{d} n_{t}-v_{t} \mathrm{~d} t\right), \\
\mathrm{d} G_{t}^{\beta \beta} & =\left(\frac{v_{t}^{\beta \beta}}{v_{t}}-G_{t}^{\beta \beta}\right)\left(\mathrm{d} n_{t}-v_{t} \mathrm{~d} t\right),
\end{aligned}
$$

where the intensities have the form

$$
\begin{aligned}
v_{t}^{\alpha \alpha} & =\left|\sqrt{\Gamma} f_{t}+\alpha_{t}\right|^{2} G_{t}^{\alpha \alpha}, \\
v_{t}^{\alpha \beta} & =\left(\sqrt{\Gamma} f_{t}+\alpha_{t}\right)\left(\sqrt{\Gamma} g_{t}^{*}+\beta_{t}^{*}\right) G_{t}^{\alpha \beta}\langle\beta \mid \alpha\rangle, \\
v_{t}^{\beta \alpha} & =\left(\sqrt{\Gamma} f_{t}^{*}+\alpha_{t}^{*}\right)\left(\sqrt{\Gamma} g_{t}+\beta_{t}\right) G_{t}^{\beta \alpha}\langle\alpha \mid \beta\rangle, \\
v_{t}^{\beta \beta} & =\left|\sqrt{\Gamma} g_{t}+\beta_{t}\right|^{2} G_{t}^{\beta \beta} .
\end{aligned}
$$

For the homodyne observation, we get

$$
\mathrm{d} G_{t}^{\alpha \alpha}=\left(\mu_{t}^{\alpha \alpha}-G_{t}^{\alpha \alpha} \mu_{t}\right)\left(\mathrm{d} q_{t}-\mu_{t} \mathrm{~d} t\right)
$$




$$
\begin{aligned}
\mathrm{d} G_{t}^{\alpha \beta} & =\left(\frac{\mu_{t}^{\alpha \beta}}{\langle\beta \mid \alpha\rangle}-G_{t}^{\alpha \beta} \mu_{t}\right)\left(\mathrm{d} q_{t}-\mu_{t} \mathrm{~d} t\right), \\
\mathrm{d} G_{t}^{\beta \alpha} & =\left(\frac{\mu_{t}^{\beta \alpha}}{\langle\alpha \mid \beta\rangle}-G_{t}^{\beta \alpha} \mu_{t}\right)\left(\mathrm{d} q_{t}-\mu_{t} \mathrm{~d} t\right), \\
\mathrm{d} G_{t}^{\beta \beta} & =\left(\mu_{t}^{\beta \beta}-G_{t}^{\beta \beta} \mu_{t}\right)\left(\mathrm{d} q_{t}-\mu_{t} \mathrm{~d} t\right),
\end{aligned}
$$

where

$$
\begin{aligned}
& \mu_{t}^{\alpha \alpha}=\left(\sqrt{\Gamma}\left(f_{t}+f_{t}^{*}\right)+\alpha_{t}+\alpha_{t}^{*}\right) G_{t}^{\alpha \alpha}, \\
& \mu_{t}^{\alpha \beta}=\left(\sqrt{\Gamma}\left(f_{t}+g_{t}^{*}\right)+\alpha_{t}+\beta_{t}^{*}\right) G_{t}^{\alpha \beta}\langle\beta \mid \alpha\rangle, \\
& \mu_{t}^{\beta \alpha}=\left(\sqrt{\Gamma}\left(f_{t}^{*}+g_{t}\right)+\alpha_{t}^{*}+\beta_{t}\right) G_{t}^{\beta \alpha}\langle\alpha \mid \beta\rangle, \\
& \mu_{t}^{\beta \beta}=\left(\sqrt{\Gamma}\left(g_{t}+g_{t}^{*}\right)+\beta_{t}+\beta_{t}^{*}\right) G_{t}^{\beta \beta} .
\end{aligned}
$$

Initially we have $G_{0}^{\alpha \alpha}=G_{0}^{\alpha \beta}=G_{0}^{\beta \alpha}=G_{0}^{\beta \beta}=1$. One can prove (135) by inserting the conditional operators $\tilde{\rho}_{t}^{\alpha \alpha}, \tilde{\rho}_{t}^{\alpha \beta}, \tilde{\rho}_{t}^{\beta \alpha}$, and $\tilde{\rho}_{t}^{\beta \beta}$ of the proposed forms into the relevant filtering equations. One can check that this leads to the given differential equations for the amplitudes $f_{t}$ and $g_{t}$, and the coefficients $G_{t}^{\alpha \alpha}, G_{t}^{\alpha \beta}, G_{t}^{\beta \alpha}$, and $G_{t}^{\beta \beta}$. For the environment taken in a coherent state our formulas reduce to the known results (see, for instance, $[3,43]$ ).

\section{Conclusion}

We derived the stochastic equation describing the conditional evolution of an open quantum system interacting with the Bose field prepared in a superposition of coherent states. We consider two schemes of measurement of the output field: photon counting and homodyne detection. Instead of methods based on the quantum stochastic calculus and the cascades system model [23], we used the collision model with the environment given by an infinite chain of qubits. We assumed that the bath qubits do not interact between themselves and they are initially prepared in the entangled state being a discrete analogue of a superposition of continuous-mode coherent states. The initial state of the compound system was factorizable. Because of the temporal correlations present in the environment, the evolution of open quantum system becomes non-Markovian. We started from the discrete in time description of the problem and obtained in the continuous time limit differential filtering equations consistent with the results published in [22,24]. We would like to stress that the presented method is more straight and intuitive than the methods described in [22,24]. It not only allows to derive the equations describing the conditional evolution of the system but also enables to find the general structure of quantum trajectories. 
Acknowledgements This paper was partially supported by the National Science Center project 2015/17/B/ ST2/02026.

Open Access This article is distributed under the terms of the Creative Commons Attribution 4.0 International License (http://creativecommons.org/licenses/by/4.0/), which permits unrestricted use, distribution, and reproduction in any medium, provided you give appropriate credit to the original author(s) and the source, provide a link to the Creative Commons license, and indicate if changes were made.

\section{References}

1. Belavkin, V.P.: A continuous counting observation and posterior quantum dynamics. J. Phys. A Math. Gen. 22, L1109 (1989)

2. Belavkin, V.P.: A posterior Schrödinger equation for continuous nondemolition measurement. J. Math. Phys. 31, 2930 (1990)

3. Barchielli, A., Belavkin, V.P.: Measurements continuous in time and a posteriori states in quantum mechanics. J. Phys. A Math. Gen. 24, 1495 (1991)

4. Carmichael, H.: An Open Systems Approach to Quantum Optics. Springe, Berlin (1993)

5. Breuer, H.P., Petruccione, F.: The Theory of Open Quantum Systems. Oxford University Press, New York (2002)

6. Barchielli, A.: Continual measurements in quantum mechanics and quantum stochastic calculus. In: Attal, S., Joye, A., Pillet, C.-A. (eds.) Lecture Notes in Mathematics, vol. 1882, pp. 207-291. Springer, Berlin (2006)

7. Gardiner, C.W., Zoller, P.: Quantum Noise. Springer, Berlin (2010)

8. Wiseman, H.M., Milburn, G.J.: Quantum Measurement and Control. Cambridge University Press, Cambridge (2010)

9. Hudson, R.L., Parthasarathy, K.R.: Quantum Ito's formula and stochastic evolutions. Commun. Math. Phys. 93, 301 (1984)

10. Parthasarathy, K.R.: An Introduction to Quantum Stochastic Calculus. Birkhäuser, Basel (1992)

11. Gardiner, C.W., Collet, M.J.: Input and output in damped quantum systems: quantum stochastic differential equations and the master equation. Phys. Rev. A 31, 3761 (1985)

12. Goetsch, P., Graham, R.: Linear stochastic wave equations for continuously measured quantum systems. Phys. Rev. A 50, 5242 (1994)

13. Barchielli, A., Paganoni, A.M.: Detection theory in quantum optics: stochastic representation. Quantum Semiclassical Opt. J. Eur. Opt. Soc. Part B 8, 133 (1996)

14. Brun, T.A.: A simple model of quantum trajectories. Am. J. Phys. 70, 719 (2002)

15. Gough, J.E., Sobolev, A.: Stochastic Schrödinger equations as limit of discrete filtering. Open Syst. Inf. Dyn. 11, 235 (2004)

16. Bouten, L., Guţă, M., Maassen, H.: Stochastic Schrödinger equations. J. Phys. A Math. Gen. 37, 3189 (2004)

17. Gough, J.E., Köstler, C.: Quantum filtering in coherent states. Commun. Stochastic Anal. 4, 505 (2010)

18. Dąbrowska, A., Staszewski, P. : Filtering equation for measurement of a coherent channel. J. Opt. Soc. Am. Part B 28, 1238 (2011)

19. Da̧browska, A., Staszewski, P.: Posterior quantum dynamics for a continuous diffusion observation of a coherent channel. J. Opt. Soc. Am. Part B 29, 3072 (2012)

20. Nurdin, H.I.: Quantum filtering for multiple input multiple output systems driven by arbitrary zeromean jointly Gaussian input fields. Russ. J. Math. Phys. 21, 386 (2014)

21. Da̧browska, A., Gough, J. E.: Belavkin filtering with squeezed light sources. Russ. J. Math. Phys. 23, 172 (2016)

22. Gough, J.E., James, M.R., Nurdin, H.I., Combes, J.: Quantum filtering for systems driven by fields in single-photon states or superposition of coherent states. Phys. Rev. A 86, 043819 (2012)

23. Gough, J.E., James, M.R., Nurdin, H.I.: Single photon quantum filtering using non-Markovian embeddings. Philos. Trans. R. Soc. A 370, 5408 (2012)

24. Gough, J.E., James, M.R., Nurdin, H.I.: Quantum filtering for systems driven by fields in single photon states and superposition of coherent states using non-Markovian embeddings. Quantum Inf. Process. 12, 1469 (2013) 
25. Dąbrowska, A.: Quantum filtering equations for system driven by non-classical fields. Open Syst. Inf. Dyn. 25, 1850007 (2018)

26. Gough, J.E., James, M.R., Nurdin, H.I.: Quantum trajectories for a class of continuous matrix product input states. New J. Phys. 16, 075008 (2014)

27. Song, H.T., Zhang, G.F., Xi, Z.R.: Continuous-mode multiphoton filtering. SIAM J. Control Optim. 54, 1602 (2016)

28. Baragiola, B.Q., Combes, J.: Quantum trajectories for propagating Fock states. Phys. Rev. A 96, 023819 (2017)

29. Attal, S., Pautrat, Y.: From repeated to continuous quantum interactions. Ann. Henri Poincaré 7, 59 (2006)

30. Pellegrini, C.: Existence, uniqueness and approximation of a stochastic Schrödinger equation: the diffusive case. Ann. Probab. 36, 2332 (2008)

31. Pellegrini, C., Petruccione, F.: Non-Markovian quantum repeated interactions and measurements. J. Phys. A Math. Theor. 42, 425304 (2009)

32. Pellegrini, C.: Existence, uniqueness and approximation of the jump-type stochastic Schrödinger equation for two-level systems. Stochastic Process Appl. 120, 1722 (2010)

33. Ciccarello, F.: Collision models in quantum optics. Quantum Meas. Quantum Metrol. 4, 53 (2017)

34. Bouten, L., Handel, R., James, M.R.: A discrete invitation to quantum filtering and feedback control. SIAM Rev. 51, 239 (2009)

35. Gross, J.A., Caves, C.M., Milburn, G.J., Combes, J.: Qubit models of weak continuous measurements: Markovian conditional and open-system dynamics. Quantum Sci. Technol. 3, 024005 (2018)

36. Dąbrowska, A., Sarbicki, G., Chruściński, D.: Quantum trajectories for a system interacting with environment in a single photon state: counting and diffusive processes. Phys. Rev. A 96, 053819 (2017)

37. Dąbrowska, A., Sarbicki, G., Chruściński, D.: Quantum trajectories for a system interacting with environment in $N$-photon state. J. Phys. A Math. Theor. 52, 105303 (2019)

38. Meyer, P.-A.: Quantum Probability for Probabilists. Lecture Notes in Mathematics, vol. 1538. Springer, Berlin (1995)

39. Attal, S.: Lecture Notes in Mathematics, vol. 1801, pp. 477-491. Springer, Berlin (2003)

40. Gough, J.E.: Holevo-ordering and the continuous-time limit for open Floquet dynamics. Lett. Math. Phys. 67, 207 (2004)

41. Pautrat, Y.: From Pauli matrices to quantum Itô formula. Math. Phys. Anal. Geom. 8, 121 (2005)

42. Fischer, K.A., Trivedi, R., Ramasesh, V., Siddiqi, I., Vučković, J., : Scattering into one-dimensional waveguides from a coherently-driven quantum-optical system. Quantum 2, 69 (2018)

43. Carmichael, H.J.: Statistical Methods in Quantum Optics, vol. 1. Springer, Berlin (2002)

Publisher's Note Springer Nature remains neutral with regard to jurisdictional claims in published maps and institutional affiliations. 